Página inicial: 383 - Página final: 392

Tipo de artículo: de investigación

\title{
Estrategia para el reconocimiento de la identidad cultural y el sentido de pertenencia de inmigrantes latinoamericanos.
}

A Strategy for the recognition of the cultural identity and the sense

of belonging of Latin American immigrants.

\begin{tabular}{|lll|}
\hline Recibido: noviembre $2015 \quad$ Revisado: abril $2016 \quad$ Aceptado: mayo 2016 \\
\hline
\end{tabular}

Por: Angie Enerieth Coronado Bohórquez ${ }^{1}$, Nidia Milena Moreno López ${ }^{2}$, Ana Carolina Torres Gómez ${ }^{3}$

\section{Resumen.}

Se hace necesario formar directivos escolares y docentes voluntarios que implementen estrategias pedagógicas que mejoren el proceso de enseñanza-aprendizaje, en el cual, su principal factor sea el rescate de la identidad cultural y el sentido de pertenencia Latinoamericano. Se presenta así, un estudio que genera insumos para la formación de docentes en habilidades cognitivas y pedagógicas para la enseñanza cultural, asesoría en el diseño de actividades didácticas para el desarrollo de dicha enseñanza, para la población inmigrante del Estado de Massachusetts- Estados Unidos.

\section{Palabras clave.}

Estrategias pedagógicas, identidad cultural, sentido de pertenecía, comunidad, inmigrantes.

\section{Abstract.}

It is necessary to train school managers and teachers volunteers, who can implement pedagogical strategies, which improve the teaching-learning process, in which its main factor is the rescue of the cultural identity and the sense of belonging in Latin America. Thus, a study, which generates inputs for teacher training in cognitive and pedagogical skills for the cultural education, the consultancy on the design of teaching activities for the development of such a teaching, for the immigrant population of the State of Massachusetts - United States, is introduced.

\section{Key words.}

Pedagogical Strategies, Cultural Identity, Sense of Belonging, Community, Immigrants.

\footnotetext{
${ }^{1}$ Psicóloga, docente investigadora Universidad Nacional Abierta y a Distancia (Unad). Medellín, Colombia. Contacto: anenerieth@gmail.com

2 Psicóloga Universidad Pedagógica Tecnológica de Colombia, Esp. Educación y Orientación Familiar FUM, PhD

(C) Psicología. Docente investigadora Universitaria (Unad). Medellin, Colombia.

${ }^{3}$ Psicóloga UAN, Docente Universitaria (Unad). Medellín, Colombia
} 


\section{Introducción.}

Esta investigación se desarrolló conceptualmente desde el enfoque Constructivista Social, teniendo en cuenta el eje central enseñanza - aprendizaje en el contexto educativo. Este tipo de aprendizaje se logra con la construcción de la estructura cognitiva propia del niño y no solamente registrando conocimientos en el cerebro (Vygotsky, 1978). Ya que desde el Constructivismo se plantea la importancia de la instrucción como método para introducir al niño/a en el mundo cultural del adulto y promover el sentido de pertenencia por sus raíces. Ya que la enseñanza no debe constituir una simple transmisión de conocimientos, por el contrario, debe establecerse como una organización de apoyo que oriente, facilite e incentive a los estudiantes en la construcción de su propio saber sin desvalorar la identidad cultural y el sentido de pertenencia Latinoamericano.

Por lo tanto, la formación de maestros voluntarios en el conocimiento de las etapas del desarrollo del niño de acuerdo a la teoría del desarrollo cognitivo, permite planear y diseñar actividades adecuadas para cada una de las edades enfocados en el rescate cultural y el sentido de pertenencia Latinoamericano en la población inmigrante. Teniendo en cuenta la importancia de un aprendizaje basado en conocimientos previos y la conexión con nuevos conocimientos para la obtención de un aprendizaje significativo Ausubel (1976).

El aprendizaje significativo, debe retomar aspectos culturales útiles para la vida diaria, aprovechando los conocimientos previos de las costumbres y la lengua de origen, reforzados con estrategias didácticas que permitan generar nuevos conocimientos culturales que contribuyan al rescate de la identidad cultural y el sentido de pertenencia del niño Latinoamericano en calidad de inmigrante en el Estado de Massachusetts.

La población inmigrante de origen hispano de los Estados Unidos especialmente los niños, tienden a tener problemas de aprendizaje debido a varios factores determinados principalmente en el uso de la lengua, la falta de identidad y el sentido de pertenencia que tengan por sus raíces, complementado con un sistema educativo que no favorece la diversidad cultural y los sentimientos de arraigo en la población inmigrante y un insuficiente apoyo en cuanto recursos humanos y económicos a las comunidades escolares hispanas (Aguilera \& Rodríguez, 2009)

Según Páez y González (2000) en su estudio "culture and social psychology", resaltan la cultura y la identidad social étnica y las define como las características culturales de un grupo o país, es decir, normas, valores, creencias y patrones de comportamiento. La identidad cultural se refiere a diferentes facetas como la auto-categorización, las actitudes y sentimientos hacia el país al que pertenece o grupo de origen. Teniendo el concepto del país al que pertenece o identificación, orgullo étnico o autoestima colectiva; (Yo soy mexicano y estoy orgulloso de ser un Latinoamericano), conocimiento de los valores del país o grupo, tradiciones, la actitud y uso de la lengua hispana o lenguas nativas, participación con los miembros del grupo y las prácticas y la evaluación de las mismas.

En los estudios sobre inmigrantes de EEUU Phinney (1996), menciona que "la autocategorización cultural y actitudes positivas hacia el grupo étnico o país de origen siguen siendo altas a través de generaciones, mientras que el conocimiento de la lengua, las 
costumbres, los valores, normas y las prácticas culturales disminuyen". Además, la relación de identidad personal y autoestima se han visto afectadas al estar inmersos en otra cultura, pero la identificación con un carácter cultural podría permanecer fuerte, incluso cuando hay poca participación cultural.

El sentido de pertenenciay de identidad se refleja en los valores, costumbres y manifestaciones culturales que se construyen y mantienen al sentirse como parte de una familia, de un grupo o de una nación (Bourdieu, 1980) En este sentido cuando el inmigrante se siente ciudadano de su país, pero no ciudadano del país receptor, se debate entre su lugar de nacimiento y su lugar de vida, porque considera que no es "ni de aquí, ni de allá" (Vargas, 2011). Por tal razón desde la misma comunidad inmigrante de origen hispano surge la necesidad de construcción de subjetividad a partir de escenarios de socialización, mediante la creación de un programa educativo sociocultural para fomentar la identidad cultural y el sentido de pertenencia Latinoamericano y dar soporte a las problemáticas que enfrentan los niños inmigrantes en el Estado de Massachusetts.

\section{Metodologia.}

Esta es una Investigación de tipo cualitativo dado que la relación entre la teoría y la investigación es abierta e interactiva. Utilizando el enfoque de investigación critico-social y como método la investigación participativa (IP), puesto que permite la generación de conocimientos a partir de la realidad que la comunidad está viviendo, al hacer parte de la misma realidad (De Miguel Díaz, 1989)

Con el fin de lograr establecer un diagnóstico sobre las necesidades que presentan los docentes en cuanto al proceso de enseñanza-aprendizaje se realiza: un proceso de observación participante que establece un acercamiento a los maestros voluntarios para observar las conductas, actitudes y necesidades que posteriormente deben plasmar en un diario de campo, por medio del cual se sistematiza las observaciones hechas durante el acercamiento en el momento de impartir sus clases. Entrevista de clasificación con preguntas abiertas y estructuradas dirigidas a los maestros voluntarios para generar un análisis de la problemática, determinando con claridad el diseño del proceso de formación de los maestros. grupo focal conformado por los maestros y directivos de la ONG, para generar espacios de interacción, análisis y discusión de problemáticas y diseño de nuevas estrategias pedagógicas para la enseñanza de la cultura Latinoamericana. Por último, se utiliza entrevistas post para la recolección de resultados del proceso de intervención y determinar el impacto que tuvo el proceso en la ONG.

Se diseñó y planteó la estrategia docente, fundamentado en el constructivismo con enfoque critico-social, con el método de investigación participante (IP) la animación socio cultural, cuyo objetivo fue el fortalecer y rescatar las costumbres y tradiciones Latinoamericanas; como también el motivar el reconocimiento de la lengua hispana y la promoción de los valores morales. Se orientó la formación de docentes en habilidades cognitivas y pedagógicas para la enseñanza cultural, el asesoramiento en diseño de actividades lúdicas y didácticas en el proceso de enseñanza- aprendizaje. Teniendo la estrategia como soporte el enfoque Constructivista, dado que en el proceso de enseñanza -aprendizaje requiere de conocimientos construidos con base a experiencias críticas que posibiliten el aprendizaje 
significativo, en el que se perciba la enseñanza como una actividad crítica y al docente como un profesional autónomo que investiga reflexionando sobre su práctica (Avila, 1992)

\section{Resultados.}

Al realizar el acercamiento a la comunidad educativa del Ministerio Infantil de la ONG, mediante la observación participante y sistematización de las conductas, actitudes y necesidades de los maestros voluntarios en el momento de impartir sus clases, reflejó que no contaban con las herramientas pedagógicas y didácticas necesarias para el desarrollo de la clase. Evidenciado en comportamientos pasivos, tono de voz bajo, inadecuado establecimiento de normas y manejo de la disciplina, conductas autoritarias y vocabulario complejo para la comprensión de los niños dentro del aula de clase, lo cual ocasionaba desmotivación por aprender acerca de la cultura y los valores morales.

De acuerdo con los resultados preliminares de la observación, se estableció la necesidad de aplicar una entrevista de clasificación mixta a los maestros voluntarios, en la que se encontró carencia de conocimientos sobre pedagogía y didáctica para el desarrollo de actividades lúdicas dentro del programa educativo cultural que presta la ONG.

\section{Programa de Formación Docente dirigido a los Maestros Voluntarios suscritos a la ONG.}

Se programó una actividad de formación docente donde se realizaron conferencias enfocadas en las temáticas de pedagogía y didáctica, en las que se destacaron las etapas del desarrollo de Piaget, el aprendizaje cultural de Vygotsky, el aprendizaje significativo de Ausubel y el Constructivismo como modelo de enseñanza. Primero se realizó una conferencia sobre estrategias en el manejo de la clase, la disciplina, el respeto, la autoridad del maestro, los tipos de inteligencia, los estilos de aprendizaje y algunas actividades lúdicas para generar motivación en los estudiantes y fomentar la construcción de aprendizaje significativo, a todos los participantes conformados por veinte (20) maestros voluntarios y dos (2) directivos.

Posteriormente se dividió a los participantes en grupos, teniendo en cuenta las edades de los estudiantes con los que están vinculados en el programa. Se socializó a cada uno de los grupos el proceso de desarrollo cognitivo del niño teniendo en cuenta las etapas del desarrollo cognitivo del niño de Piaget (1964), comenzando con los maestros que manejan los niños que están entre los 0 a 2 años, enfocándose en la etapa senso- Motora. Posteriormente con los maestros de niños de 3 a 5 años profundizando en la etapa pre operacional. Finalmente, con la etapa operacional concreta, dirigida a los maestros encargados de los niños de 6 a 12 años.

Posteriormente se dio paso a la formación docente enfocada en el aprendizaje cultural y significativo desde los fundamentos teóricos de Vygotsky y Ausubel, siendo el modelo de Constructivismo un factor relevante de estudio, lo que conllevo a reflexionar en la importancia de la aplicación de métodos de enseñanza adecuados que generen un impacto positivo en el proceso de enseñanza-aprendizaje que conduzcan a las metas propuestas en el programa sociocultural de la ONG. 
La respuesta al programa de formación docente fue receptiva por parte de los maestros y directivos, evidenciado en los resultados de la entrevista post, donde se evaluó la influencia y el impacto de la formación docente.

\section{Grupos Focales, un Espacio de Discusión en el Diseño de Estrategias de Enseñanza}

El diseño de grupos focales conformados por los maestros voluntarios y los directivos escolares divididos en grupos de acuerdo a las edades de los estudiantes promovió el análisis de las necesidades existentes, creando un espacio de discusión y opinión para diseñar nuevas estrategias pedagógicas y didácticas que contribuyeran al reconocimiento de la identidad cultural y el sentido de pertenencia Latinoamericano en los niños inmigrantes del Estado de Massachusetts adscritos al programa de la ONG, considerando como principales elementos la animación socio cultural, el reconocimiento de la lengua hispana y la promoción de los valores morales.

Se diseñó una estrategia pedagógica con herramientas y actividades lúdicas enfocadas en la expresión corporal y el uso de la lengua hispana, teniendo en cuenta los contenidos de los valores morales, las costumbres y tradiciones latinoamericanas constituidas por los trajes autóctonos de cada país de los participantes, los platos típicos, las danzas y la música, además de los deportes en los que se destacan. Se implementó el teatro y la danza como actividades de animación sociocultural donde la participación de los padres de familia y los maestros fue necesaria para apoyar la estrategia.

\section{El proceso de evaluación de los alumnos suscritos al programa educativo cultural de la Iglesia Cristiana Celebración- ONG.}

Se determinó que en todo proceso de aprendizaje la evaluación debe jugar un papel importante, ya que es útil para analizar el impacto de la Acción Psicosocial en el proceso de enseñanza-aprendizaje y en la motivación de los actores. De la misma manera la evaluación permite la toma de decisiones en el proceso de formación y en el desarrollo de los materiales y métodos de instrucción, partiendo del análisis en el método de evaluación implementado por la ONG, el cual fue rediseñado para mayor efectividad, induciendo a la evaluación formativa donde además de dar "dinero didáctico" a los estudiantes por su participación en el programa cultural, se realizó un evento cultural donde se pudo utilizar ese "dinero didáctico" para incrementar la motivación en los actores del proceso.

Desde el enfoque Constructivista Social se determina que el eje central es la construcción activa de conocimiento, dando como resultado un aprendizaje significativo, el cual hace referencia a que el conocimiento construido a partir de las herramientas dadas por el maestro voluntario sea utilizado en la vida cotidiana dentro de las tradiciones y las costumbres Latinoamericanas, los valores morales y la lengua hispana. Por medio de la evaluación se puede evidenciar si realmente es un aprendizaje significativo, lo cual se tiene en cuenta para hacer el siguiente análisis de resultados.

Es de resaltar que, en la organización, el Ministerio Infantil cuenta con un sistema de evaluación denominado sistema Economía de Fichas donde se clasifican diferentes rangos: 
Tabla 1. Clasificación del Sistema de Evaluación por Economia de Fichas

\begin{tabular}{ll}
\hline Rangos & Equivalente en dinero didáctico \\
\hline Reprobado & 1 dólar didáctico \\
Regular & 5 dólares didácticos \\
Bueno & 10 dólares didácticos \\
Muy bueno & 15 dólares didácticos \\
Excelente & 20 dólares didácticos
\end{tabular}

Tabla 1. En esta tabla se presenta el proceso de evaluación docente denominado sistema de economía de fichas donde se clasifican en diferentes rangos.

Tabla 2. Criterios de evaluación

\section{Criterios de evaluación}

E1 trabajo en clase y participación.

El trabajo en equipo.

El comportamiento.

Asistencia

Tabla 2. Esta tabla representa los criterios que se evalúan en el proceso de enseñanzaaprendizaje desarrollado en el programa educativo sociocultural.

Teniendo en cuenta los grupos de edades, los criterios y rangos de evaluación; se tomaron los resultados del proceso hecho a cada uno de los grupos de estudiantes participantes del programa cultural antes de la aplicación de la estrategia de formación docente y se hizo un contraste con los resultados de la evaluación después de la aplicación de dicha estrategia, para determinar la influencia en la motivación y el rendimiento académico de los actores del proceso. Para lo cual se expresa en el siguiente cuadro.

Tabla 3. Resultados de la evaluación y la influencia del proceso de formación docente

\begin{tabular}{lll}
\hline \hline Grupos & $\begin{array}{l}\text { Resultados de la evaluación del periodo } \\
\text { Pre (antes del proceso de formación } \\
\text { docente) }\end{array}$ & $\begin{array}{l}\text { Resultados de la evaluación en el } \\
\text { periodo Post (durante y después del } \\
\text { proceso de formación docente) }\end{array}$ \\
\hline \hline 0-2 años & $\begin{array}{l}\text { El trabajo en clase y } \\
\text { participación: } 10 \text { dólares } \\
\text { El trabajo en equipo: } 5 \text { dólares } \\
\text { El comportamiento: } 10 \text { dólares } \\
\text { Asistencia: } 10 \text { dólares }\end{array}$ & $\begin{array}{l}\text { El trabajo en clase y } \\
\text { participación: 20 dólares } \\
\text { El trabajo en equipo: 20 dólares } \\
\text { El comportamiento: } 15 \text { dólares } \\
\text { Asistencia: 20 dólares }\end{array}$ \\
\hline
\end{tabular}




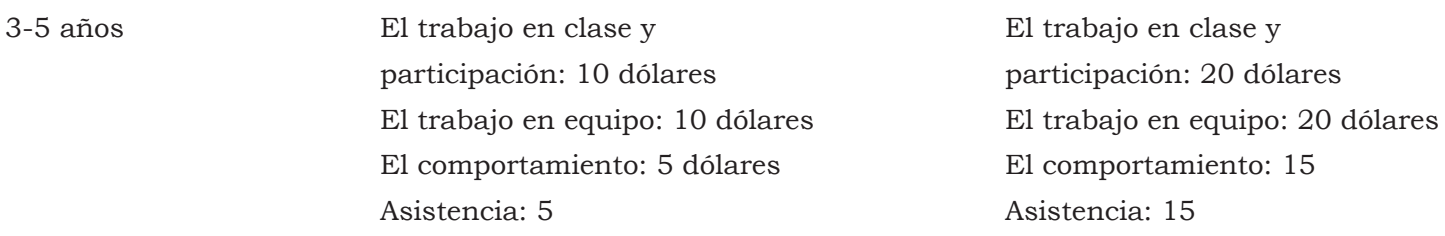

\begin{tabular}{|c|c|c|}
\hline \multirow[t]{5}{*}{ 6-8 años } & El trabajo en clase y & El trabajo en clase y \\
\hline & participación: 15 dólares & participación: 20 dólares \\
\hline & El trabajo en equipo: 15 dólares & El trabajo en equipo: 20 dólares \\
\hline & El comportamiento: 10 dólares & El comportamiento: 15 dólares \\
\hline & Asistencia: 10 dólares & Asistencia: 20 dólares \\
\hline \multirow[t]{5}{*}{ 9-10 años } & El trabajo en clase y & El trabajo en clase y \\
\hline & participación: 15 dólares & participación: 20 dólares \\
\hline & El trabajo en equipo: 15 dólares & El trabajo en equipo: 20 dólares \\
\hline & El comportamiento: 10 dólares & El comportamiento: 15 dólares \\
\hline & Asistencia: 15 dólares & Asistencia: 20 dólares \\
\hline \multirow[t]{5}{*}{ 11-12 años } & El trabajo en clase y & El trabajo en clase y \\
\hline & participación: 15 dólares & participación: 20 dólares \\
\hline & El trabajo en equipo: 15 dólares & El trabajo en equipo: 20 dólares \\
\hline & El comportamiento: 15 dólares & El comportamiento: 15 dólares \\
\hline & Asistencia: 15 dólares & Asistencia: 20 dólares \\
\hline
\end{tabular}

Tabla 3. Presenta los resultados de la evaluación antes, durante y después del proceso de formación docente, de la misma manera muestra la influencia de dicho proceso en el rendimiento académico.

De acuerdo con la tabla, se evidencia que en el periodo Pre el rendimiento académico en cada uno de los grupos fue inferior al periodo Post. Lo que permite evidenciar la eficacia del proceso de formación docente, ya que el diseño de actividades adecuadas en cada una de las edades de los estudiantes y el conocimiento de métodos y estrategias de enseñanza, fueron útiles en el diseño e implementación de las actividades lúdicas con propósitos culturales, logrando un gran impacto que se reflejó en la motivación y el interés de los estudiantes por participar en las actividades culturales y de sentido de pertenencia; como en el aprendizaje y la práctica de la lengua hispana que se convirtió en una de las principales atracciones de los participantes del programa. Dando como resultado final el fortalecimiento y rescate de las costumbres y tradiciones Latinoamericanas.

Los maestros voluntarios manifestaron que al contar con herramientas pedagógicas y el apoyo de los padres de familia se sentían más motivados para el desarrollo de las actividades relacionadas con el reconocimiento de la lengua hispana y la promoción de los valores morales en los estudiantes, incorporando las costumbres y tradiciones propias de los países de origen. 
Se cumple con el propósito de la investigación dando formación a los maestros voluntarios, donde cada uno de ellos identifica y reconoce habilidades cognitivas y pedagógicas para la enseñanza cultural, teniendo como base el modelo del constructivismo donde la enseñanza se considera una actividad crítica y al docente un actor autónomo que investiga reflexionando sobre su práctica e implementando dichas habilidades a la hora de impartir sus clases, teniendo en cuenta la edad y características cognitivas de cada uno de los actores (Avila, 1992) Los maestros logran mediar entre los conocimientos previos y los nuevos para construir aprendizaje cultural significativo, usando herramientas y estrategias de enseñanza enfocadas en la cultura latinoamericana fomentando la identidad cultural, el sentido de pertenencia por sus raíces y los valores morales.

Con la formación del grupo focal se brindó asesoramiento a la comunidad educativa y se generaron espacios de discusión que cumplieron el objetivo de promover, facilitar y mantener los procesos de comunicación entre los grupos de maestros y directivos, fundamentados en la reflexión y el intercambio de prácticas transformadoras de su quehacer.

Finalmente, la animación socio cultural permitió fortalecer la identidad de los inmigrantes, su sentido de pertenencia y el mejoramiento de la convivencia mediante la participación en el diseño y solución de los problemas sociales y culturales que les afecta como comunidad (Merino, 1997)

\section{Conclusiones.}

Es de destacar el trabajo hecho con los docentes, en los cuales se evidenció la importancia de conocer metodologías que se requieren en el acompañamiento pedagógico para contribuir en la motivación en el proceso de enseñanza-aprendizaje, posibilitando el mejoramiento de la convivencia y favoreciendo que el aprendizaje sea realmente significativo para los estudiantes.

Estos resultados reflejan la importancia de brindar capacitación constante a los docentes, para que puedan lograr transformaciones significativas, desde la implementación de una pedagogía para la enseñanza sociocultural en donde se reconozcan métodos didácticos y creación de ambientes favorables al aprendizaje.

El diseño e implementación de actividades y métodos de enseñanza enfocados en el estudio y en el uso de la lengua hispana, permite minimizar las dificultades que se presentan en los procesos de enseñanza-aprendizaje de los niños inmigrantes o hijos de inmigrantes suscritos al programa sociocultural que desarrolla la ONG.

Teniendo en cuenta que el sentido de pertenencia de la población inmigrante por sus países de origen ha disminuido y aún más en los niños que llegaron a edad temprana; la comunidad inmigrante perteneciente a la Iglesia cristiana ONG mediante la implementación del programa educativo sociocultural, logró motivar a los maestros voluntarios, padres de familia y niños en el proceso enseñanza- aprendizaje de la lengua hispana y en el rescate de las costumbres tradiciones, valores y creencias con las cuales se sienten identificados y orgullosos de sus países de origen. 
Se cumplió con el propósito de la investigación con los docentes voluntarios, con el desarrollo de las habilidades cognitivas y pedagógicas para la enseñanza sociocultural para el fortalecimiento de la identidad cultural, el sentido de pertenencia por sus raíces y los valores morales.

Se brindó asesoramiento a la comunidad educativa, generando espacios de discusión que cumplieron el objetivo de promover, facilitar y mantener los procesos de comunicación entre los grupos de maestros y directivos, fundamentados en la reflexión y el intercambio de prácticas transformadoras de su quehacer. 


\section{Referencias bibliográficas.}

Aguilera, S., \& Rodríguez, M. (2009). La educación de los hijos de inmigrantes mexicanos en Estados Unidos Mexico DF. México.

Alfonso, I., \& González, T. (1991). Proceso de enseñanza-aprendizaje: Algunas características y particularidades. Madrid: Visor.

Avila, A. R. (1992). Cómo Aprende y Cómo Enseña el Docente. Seminario (Ponencia por Colombia). Chile.

Bourdieu, P. (1980). L’identité et la représentation. Actes de la recherche en sciences sociales, 35(1), 6372.

Corbetta, P. (2010). Metodología y técnicas de investigación social. Madrid: McGraw.

De Miguel Díaz, F. M. (1989). Modelos de investigación sobre organizaciones educativas. Revista de investigación educativa, RIE, 7(13), 21-56.

Merino, J. F. (1997). Animación sociocultural en la educación social. Exigencias formativas para el educador social. Revista Complutense de Educación, 8(1).

Páez, D., \& González, J. L. (2000). Culture and social psychology. Psicothema, 6-15. Obtenido de http:/ / www.redalyc.org/articulo.oa?id=72796002

Vargas, M. D. (2011). Ciudadanía e inmigración: La nueva frontera entre la pertenencia y la exclusión. LiminaR. Estudios Sociales y Humanísticos, 48-56. Obtenido de http:// www.redalyc.org/articulo.oa?id=74522310005 